\title{
Pacemapping the Triangle of Koch Reveals Unusual Atrioventricular Nodal Fast Pathway Inputs in Pediatric Patients
}

\author{
ANTHONY C. MCCANTA, MD ${ }^{1,2}$, MICHELLE E. KIGER, MD ${ }^{3,4}$, LAURA L. PYLE, $\mathrm{PhD}^{3,4}$, \\ D. MARTIN RUNCIMAN, MBBS ${ }^{3,4}$ and KATHRYN K. COLLINS, MD ${ }^{3,4}$ \\ ${ }^{1}$ Children's Hospital Orange County, Orange, CA \\ ${ }^{2}$ University of California, Irvine, School of Medicine, Department of Pediatrics, Orange, CA \\ ${ }^{3}$ University of Colorado Denver School of Medicine, Department of Pediatrics, Aurora, CO \\ ${ }^{4}$ Children's Hospital Colorado, Aurora, CO
}

\begin{abstract}
Pacemapping the triangle of Koch determines the location of atrioventricular $(A V)$ nodal fast pathway inputs in adults. This study aims to evaluate the technique in pediatrics. A retrospective, single-center study of pediatric patients who underwent ablation for atrioventricular nodal reentrant tachycardia (AVNRT) was performed. Pacemapping was performed from the posteroseptal (PS), midseptal (MS), and anteroseptal (AS) locations. The stimulation to His bundle (SH) times were recorded in milliseconds (ms). The usual fast pathway position was defined as progressive decrease from PS to MS to AS. An abnormal response was defined as $S H$ time in the MS or PS location that was less than that from the AS. Fifty-six ablation procedures were reviewed in 56 patients. The usual fast pathway position was documented in 53 of 56 patients (94.6\%), with SH times measured in the PS of $97 \mathrm{~ms}$ (63-189 ms), MS of 82 (50-166 ms), and AS of 71 (35-161 ms). Unusual fast pathway inputs were observed in three patients (5.4\%). Seven of 56 patients (12.5\%) sustained transient AV nodal injury that resolved within minutes. Unusual fast pathway inputs were observed in $5.4 \%$ of pediatric patients undergoing slow pathway ablation for AVNRT. Transient AV nodal injury was documented in $12.5 \%$ of cryoablations. No patients with unusual fast pathway inputs had transient AV nodal injury.
\end{abstract}

KEYWORDS. Atrioventricular nodal reentrant tachycardia, catheter ablation, congenital heart disease, pediatrics, pace mapping the fast pathway.

\section{Introduction}

Atrioventricular nodal reentrant tachycardia (AVNRT) is the most common cause of supraventricular tachycardia (SVT) in adults and adolescents. In children, AVNRT increases as a cause of SVT with increasing age, such that it is rare in neonates and common in adolescents. ${ }^{1}$

The authors report no conflicts of interest for the published content. Manuscript received July 6, 2016, Final version accepted August 15, 2016.

Address correspondence to: Anthony C. McCanta, MD, Clinical Assistant Professor of Pediatrics, University of California, Irvine, Children's Hospital Orange County, 1201 W. La Veta Ave., Orange, CA 92886. E-mail: amccanta@choc.org
Although catheter ablation of AVNRT, with either radiofrequency energy or cryoenergy, is frequently utilized as a first-line approach to treatment, complete atrioventricular (AV) nodal block has been reported in up to $0.5-2 \%$ of radiofrequency cases, ${ }^{2-4}$ but it has yet to be documented using cryoablation. Unfortunately, it has been difficult to predict which patients would suffer this complication, although permanent injury to the normally anteriorly positioned AV nodal fast pathway inputs or the compact AV node itself is the likely mechanism. In 2001, Delise et al. ${ }^{5}$ described a technique to identify the location of the AV nodal fast pathway inputs by pacing from an ablation catheter in posteroseptal (PS), midseptal (MS), and anteroseptal (AS) locations in 
the triangle of Koch before ablation and measuring the time from stimulation to the His bundle electrogram. Delise and colleagues ${ }^{6}$ then applied this technique to 422 consecutive adult patients with a normal heart structure, and they reported no AV block using radiofrequency energy in the group in which the pace mapping was performed. The utility of this technique has not yet been reported in pediatrics or congenital heart disease. The purpose of this study is to evaluate for unusual AV nodal fast pathway location using the pace-mapping technique in pediatric patients, and to determine if the presence of unusual AV nodal fast pathway inputs influences the safety and efficacy of AVNRT ablation in this population.

\section{Materials and methods}

This study was approved by the Colorado Multiple Institutional Review Board. This was a retrospective singlecenter chart review of all patients who underwent electrophysiology study and ablation for AVNRT from November 2011 to December 2013. During this time period, the electrophysiology laboratory at the Children's Hospital Colorado used the pace-mapping technique of Delise et $\mathrm{al}^{5,6}$ as part of the standard technique of electrophysiology study before slow pathway ablation for AVNRT. Patients were included in the study if the patient 1) was between 4 years and 20 years of age; 2) had diagnosis of inducible AVNRT or presumed AVNRT at the time of invasive study; 3 ) had complete pace mapping (defined below); and 4) had undergone ablation for AVNRT. Demographic information was collected including age, gender, weight, and body surface area. Electrophysiologic history was also collected including prior ablations, other dysrhythmic substrates, cycle length of induced tachycardia, and mechanism of AVNRT (typical or atypical). Patients with congenital heart disease with atypical AV conduction systems including primum atrial septal defects, AV septal defects, or any anatomic variant with AV discordance including $l$-transposition of the great arteries were excluded from the study.

A four-catheter electrophysiology study was performed in all cases with a catheter in the high right atrium (HRA), the His bundle location at the AS tricuspid valve annulus, a decapolar catheter in the coronary sinus, and a pacing catheter in the right ventricular apex. A threedimensional electroanatomic mapping system (ESI Velocity, St. Jude Medical, St. Paul, MN, USA) was utilized in all cases. Atrial overdrive, atrial extrastimulus, ventricular overdrive, and ventricular extrastimulus pacing protocols were performed at baseline. If SVT was not induced at baseline, then the pacing protocols were repeated on isoproterenol. The substrates for AVNRT were defined as dual AV nodal physiology with a $\geq 50$-ms jump in atrial-His bundle time with a 10-ms decrement in atrial extrastimulation or sustained slow pathway conduction with PR interval greater RR interval. ${ }^{7}$ Presumed AVNRT was defined as history of documented SVT without inducible SVT after the presence of any dysrhythmic substrate including a concealed accessory pathway was ruled out during electrophysiology study.

\section{Pace mapping}

Pace mapping was conducted in all patients as per the technique described by Delise et al. ${ }^{5,6}$ The HRA catheter was removed, and the ablation catheter was advanced from the right femoral vein into the right atrium. The ablation catheter was then placed within the triangle of Koch. The ablation catheter was then positioned in PS, MS, and AS locations respectively (Figure 1), and pacing was performed from the catheter tip at a paced cycle length of $600 \mathrm{~ms}$, when the sinus cycle length was $>600 \mathrm{~ms}$, or at least $50 \mathrm{~ms}$ less than the sinus cycle length if it was $<600 \mathrm{~ms}$. The ablation catheter position for each pacing site was marked and stored in each location via the threedimensional electroanatomical mapping system as shown in Figure 2 (ESI Velocity, St. Jude Medical, St. Paul, MN, USA). The time from stimulation on the ablation catheter to the His bundle electrogram was measured at any given site if three or more consecutive stimuli captured the atrium and produced His bundle and ventricular conduction. In regards to the stimulation to His time, absolute stimulation to His intervals were collected as well as the stimulation to His interval in each location as it relates to the baseline sinus cycle length before pace mapping, and the paced cycle length of the pace mapping. A usual response was considered progressive decrease in the stimulation to His time from PS to MS to AS. An abnormal response indicating unusual AV nodal fast pathway inputs was defined as any condition in which the stimulation to His time measurement in the AS was not less than either the stimulation to His time measurement in either of the other positions (MS or PS).

\section{Ablation}

Ablation was then performed in the PS or MS locations. Cryoablation with a 6-mm-tip catheter was used as the first-line energy source, with radiofrequency as the second line, as per the protocol in our laboratory, with the three to six lesions of $4 \mathrm{~min}$ each placed while constantly evaluating the atrial-His interval for prolongation during each lesion.

Ablation outcomes were collected including procedural success, defined as the inability to induce AVNRT at the conclusion of the procedure or as elimination of evidence of the substrate for AVNRT in cases where AVNRT was not inducible before ablation. Empiric AV nodal modification was performed when the diagnosis of presumed AVNRT was made.

Complications were recorded including any significant change in $\mathrm{AV}$ nodal conduction including atrial-His time prolongation, and first-degree, second-degree, and third degree heart block, which were observed during ablation.

\section{Statistical analysis}

Continuous variables were assessed for distribution and non-parametric techniques and descriptive statistics were used for variables with evidence of non-normality. 


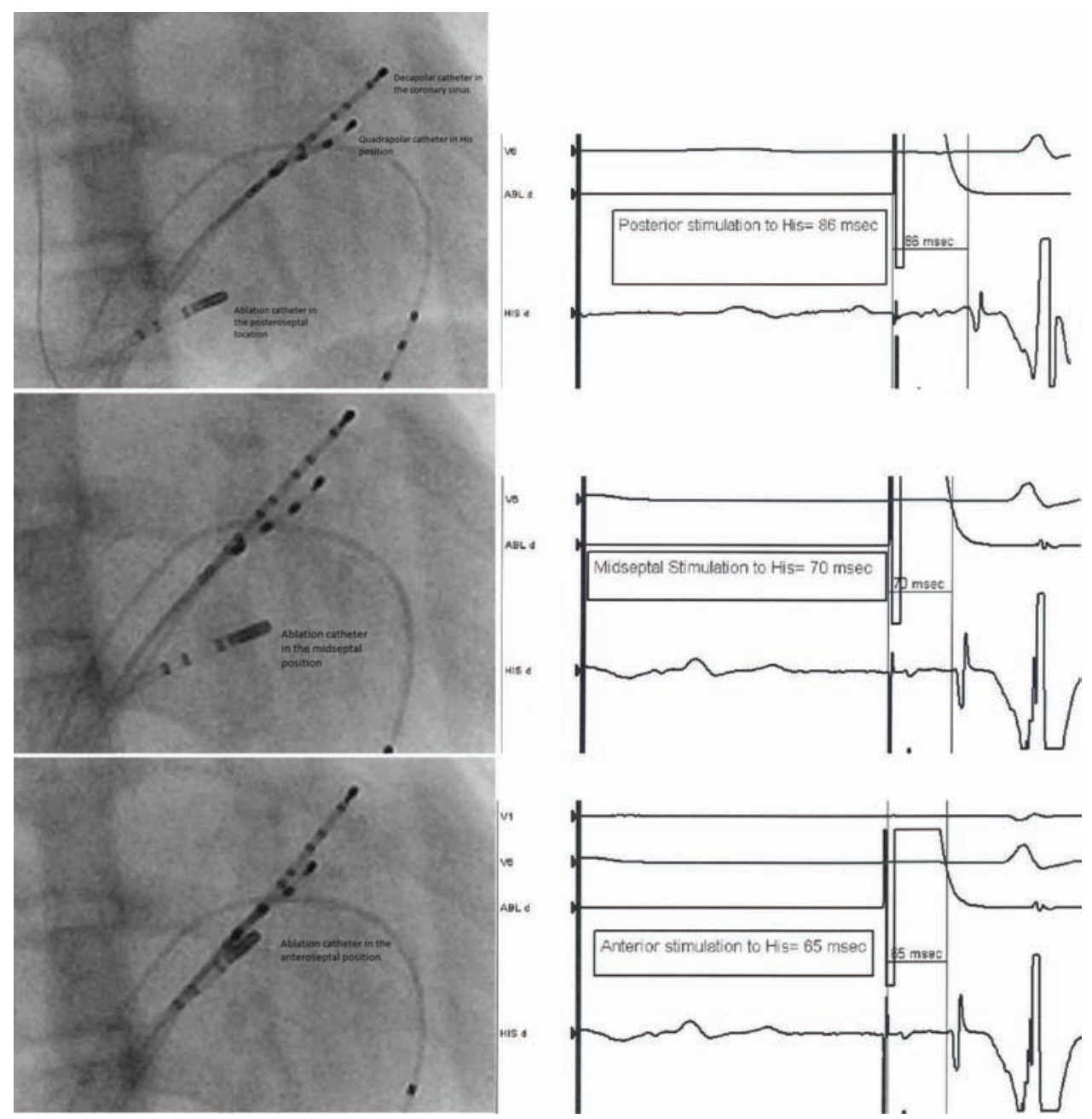

Figure 1: Right anterior oblique fluoroscopic images of posteroseptal (PS), midseptal (MS), and anteroseptal (AS) pacing locations from the ablation catheter.

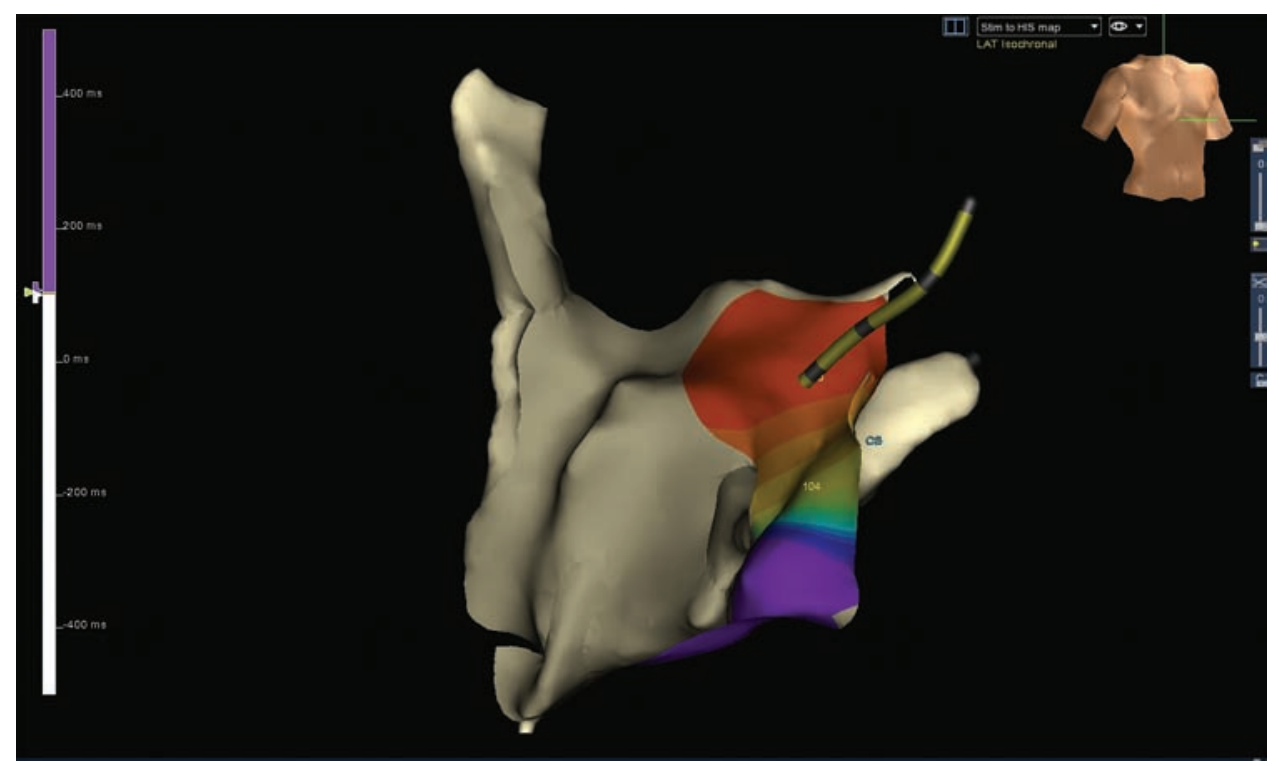

Figure 2: Right anterior oblique (RAO) projection of a three-dimensional electroanatomic pace map (ESI Velocity, St. Jude Medical, St. Paul, MN) of the triangle of Koch demonstrating the usual atrioventricular nodal fast pathway location with decreasing stimulation to His $(\mathrm{SH})$ time from posterior to anterior. Red: $\leq 99$ ms; yellow: 104 ms; purple: $\geq 115$ ms. 
Associations between categorical variables were assessed with the chi-square test or the Fisher exact test, as appropriate. Percentiles were used instead of standard deviations because of skewed distributions.

\section{Results}

\section{Demographics}

Table 1 summarizes the patient characteristics, arrhythmias, and procedural data. Congenital heart disease was present in three patients $(5.4 \%)$ and the diagnoses included one patient with surgically repaired atrial septal defect and pulmonary valve stenosis, one patient with surgically repaired patent ductus arteriosis, and one patient with unrepaired mild left-sided obstructive lesions consisting of double orifice mitral valve and a bicuspid aortic valve with mild aortic valve stenosis.

Table 1: Patient characteristics, arrhythmias, and procedural data

\begin{tabular}{lc}
\hline N (patients) & 56 \\
\hline Age (median, years) & $14.6(6.2-19.8)$ \\
Weight (median, kilograms) & $54.0(18.6-91)$ \\
Body surface area (median, meters squared) & $1.6(0.7-2.1)$ \\
AVNRT induced (patients) & $50(89.2 \%)$ \\
$\quad$ Typical (slow-fast) & $48(96 \%)$ \\
$\quad$ Atypical (fast-slow) & $2(4 \%)$ \\
Presumed AVNRT & $6(10.7 \%)$ \\
Successful slow pathway ablation (patients) & $56(100 \%)$ \\
Additional dysrhythmia observed (patients) & $4(7.1 \%)$ \\
$\quad$ Orthodromic atrioventricular reentrant & $3(5.4 \%)$ \\
$\quad$ tachycardia with concealed accessory & \\
$\quad$ pathway & \\
Permanent Form of junctional & $1(1.7 \%)$ \\
$\quad$ reciprocating tachycardia & \\
Unusual AV nodal fast pathway inputs & $3(5.4 \%)$ \\
$\quad$ patients) & $139(94-204)$ \\
Procedure time (minutes) & $2(0-22)$ \\
Fluoroscopy time (minutes) &
\end{tabular}

AV: atrioventricular; AVNRT: Atrioventricular nodal reentrant tachycardia.

\section{Procedural data}

Procedural data are presented in Table 1. Cryoablation was successful for all 56 patients. Radiofrequency was not utilized.

Pace mapping of the triangle of Koch was performed in all 56 patients included in the study. Stimulation to His times for all 56 patients are presented in Table 2.

An unusual fast pathway location was observed in three of the 56 patients $(5.4 \%)$, with the arrangement of the unusual fast pathway being MS in all three instances.

\section{Transient AV nodal injury and follow-up}

Seven patients (12.5\%) had intermittent AV nodal injury that resolved with the early termination of the cryoablation lesion. The AV node injury was transient thirddegree heart block in one patient, transient second-degree heart block in four patients, and atrial-His prolongation in two patients that prompted early termination of the lesion. None of the patients that had transient AV nodal damage had an unusual fast pathway location (Table 3).

\section{Discussion}

This study documents unusual AV nodal fast pathway inputs in $5.4 \%$ of pediatric and adolescent patients undergoing slow pathway modification for AVNRT. This finding may have important implications as to the safety and success of ablation in young patients. Although none of the patients in this study with unusual AV nodal inputs had evidence of transient AV nodal injury, the finding of unusual fast pathway location may prompt more diligence and care during the ablation, especially if the fast pathway is found to be in the posterior location. This is the first study documenting unusual fast pathway location in the pediatric and adolescent population.

In 2005, Delise et al. ${ }^{5}$ published a large study of 909 adult patients with structurally normal hearts undergoing catheter ablation for AVNRT in which a novel pace mapping technique was used to locate the AV nodal fast pathway inputs in the right atrium and thus avoid ablation in that region. ${ }^{6}$ A prior paper from the same

Table 2: Stimulation to His bundle recording time for all 56 patients (ms)

\begin{tabular}{lccccccc}
\hline Variable & Mean & Standard deviation & Median & Minimum & 25th percentile & 75th percentile & Maximum \\
\hline Posteroseptal & 99 & \pm 24.8 & 97 & 63 & 80 & 116 \\
Midseptal & 84 & \pm 23.4 & 82 & 50 & 67 & 98 \\
Anteroseptal & 72 & \pm 23.7 & 71 & 35 & 56 & 166 \\
\hline
\end{tabular}

Table 3: Cross-tabulation of unusual atrioventricular nodal position and evidence of atrioventricular nodal damage related to ablation

\begin{tabular}{lccc}
\hline Unusual position of fast pathway inputs & \multicolumn{2}{c}{ Evidence of atrioventricular nodal damage related to ablation during procedure } \\
\cline { 2 - 3 } & No & Yes & Total \\
\hline No & $46(82.14 \%)$ & $0(12.5 \%)$ & $53(94.64 \%)$ \\
Yes & $3(5.36 \%)$ & $7(0.0 \%)$ & $3(5.36 \%)$ \\
Total & $49(87.5 \%)$ & $7(12.5 \%)$ & 56 \\
\hline
\end{tabular}


group describes the technique, which was applied to 422 consecutive patients undergoing AVNRT ablation, and AV block was not observed in the 422 patients compared with seven $(1.4 \%)$ of the 487 earlier consecutive patients in which the pace-mapping technique was not used. Thus, the authors concluded that pace mapping the triangle of Koch reduces the risk of AV block in AVNRT ablation.

As a plausible biological explanation for the findings, the authors have shown that 38 of 422 patients (9\%) in whom the pace-mapping technique was utilized had abnormally positioned AV nodal fast pathways, including 33 patients $(7.8 \%)$ with MS fast pathway inputs and five $(1.2 \%)$ with posterior or absent fast pathway inputs. Because the current technique for AVNRT ablation involves the application of energy to the AV nodal slow pathway inputs which are usually located in the posterior triangle of $\mathrm{Koch}^{8,9}$ the finding that $1.2 \%$ of patients had posteriorly positioned fast pathway inputs has profound implications for ablation in that location. Although a causal relationship is not specified, the $0.5-2 \%$ AV block reported in previous studies not employing the pacemapping technique, ${ }^{2-4}$ including Delise et al.'s $1.4 \%$ of $\mathrm{AV}$ block in the group in which pace mapping was not utilized, appears to coincide with the $1.2 \%$ of posterior or absent AV nodal fast pathway inputs reported in the study.

Inadvertent AV block has been reported in the ablation of AVNRT in pediatric patients at a similar rate to the adult series. ${ }^{10}$ Because the implications of inadvertent $\mathrm{AV}$ block in previously healthy children may be profound, including the potential need for lifelong pacing involving multiple generator change procedures and the possible morbidity and even mortality from lead extractions, many pediatric and congenital heart disease centers sought ways to mitigate the risks of AVNRT slow pathway ablation. Several centers, including ours, use cryoablation as the first-line energy source, as permanent AV block has not been reported with cryoablation for AVNRT. Despite encouraging initial results, ${ }^{11}$ the mid- and long-term recurrence rates for cryoablation in a pediatric population ranges from $6.5 \%$ to $27.7 \%$, with recurrence occurring as late as 2 years after the procedure. ${ }^{12,13}$

Other centers have proposed advanced techniques to map the triangle of Koch, which are used in conjunction with modern three-dimensional electroanatomical mapping systems to identify the appropriate targets of ablation. ${ }^{14,15}$ These techniques, although elegant, do not address the rare situations of unusual fast pathway inputs, and the potential for increased risk of AV nodal damage with ablation in areas of unusual fast pathway. These techniques were not employed in our study.

The pace-mapping technique described by Delise et al. in adults takes only a few minutes to perform, and is interpreted by measurement of the stimulation from the ablation catheter to the His-bundle electrogram. This measurement is performed in real time during the procedure, and it can be performed multiple times during the procedure to validate any abnormal findings. Once the location of the fast pathway inputs is determined, this

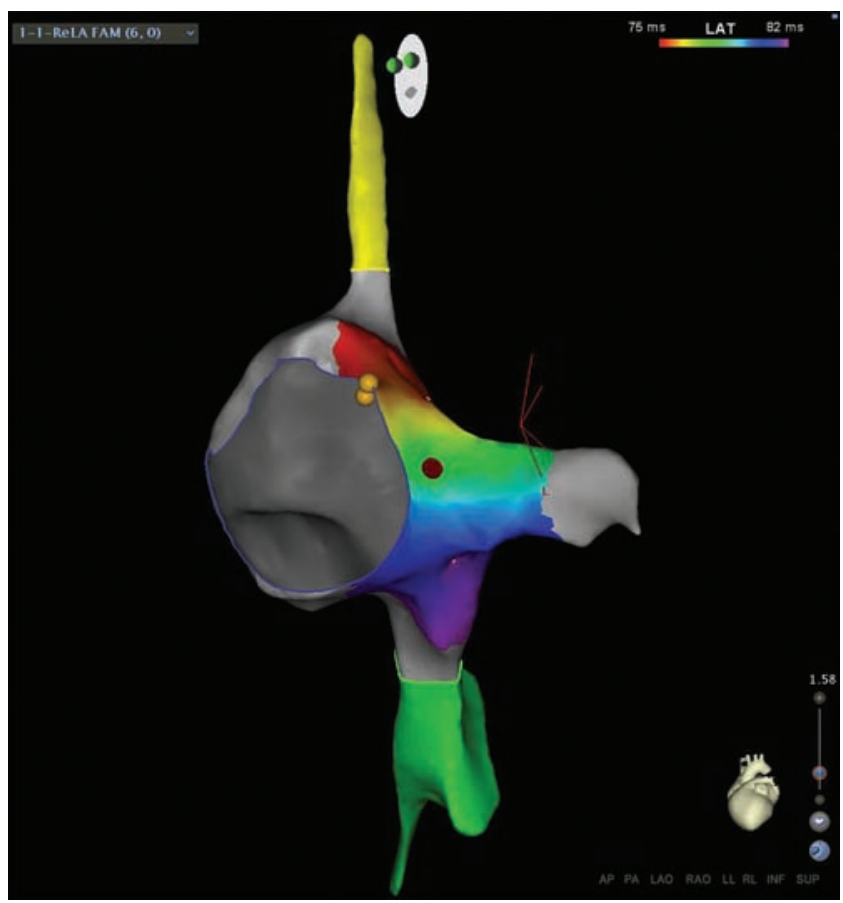

Figure 3: Left anterior oblique (LAO) projection of threedimensional electroanatomic pace map for the triangle of Koch using the Carto 3 system (Biosense Webster, Diamond Bar, CA) demonstrating usual fast pathway location and ablation in the midseptal location, posterior to the fast pathway and the His bundle. Red: $\leq 75 \mathrm{~ms}$; purple: $\geq 82 \mathrm{~ms}$; yellow dot: location of the recorded his bundle electrograms; red dot: location of successful ablation lesion.

can be saved on a three-dimensional electroanatomical mapping system (Figure 3), and ablation of the slowpathway inputs can be nearly immediately performed. When abnormal fast pathway inputs were discovered with this technique, our degree of caution when watching the atrial-His interval during cryoablation was increased, and cryoablation was terminated earlier. For operators who use radiofrequency as the first-line energy for ablation, the finding of unusual fast pathway inputs may prompt a change to cryoablation as the first-line energy source to improve the safety of the ablation. ${ }^{16}$

\section{Limitations}

This is a retrospective, single-center, descriptive study with the inherent limitations of small sample size and no comparison group. Because no permanent AV injury was observed, a safety benefit from this technique was not shown. Because no patients in this study demonstrated PS AV nodal fast pathway location, the risk of transient AV nodal injury during cryoablation in the PS location for these patients could not be assessed. Significant overlap is observed in the mean and median stimulation to His times of all patients, such that the absolute value at any one location cannot predict the pacing location. Pace mapping the triangle of Koch was also influenced by the autonomic state of the patient, and in several patients the stimulation to His time was very long, 
indicating either autonomically mediated slow conduction through the AV nodal fast pathway inputs, or potentially engagement of the $\mathrm{AV}$ nodal slow pathway inputs at the paced cycle length. As such, the absolute stimulation to His time in any one location is not as significant as the relative decrease from posterior to mid to anterior in determining the location of the AV nodal fast pathway inputs.

\section{Conclusions}

Knowledge of unusual AV nodal fast pathway inputs may have implications for the success and safety of slow pathway input ablation in pediatric patients. Although the majority of patients have a decrease in stimulation to His conduction times from posterior to anterior within the triangle of Koch, this study describes the abnormal location of the $\mathrm{AV}$ nodal fast pathway inputs in $5.4 \%$ of patients undergoing AVNRT ablation in a pediatric and congenital heart disease population. Although the findings did not influence the success rate of ablation or the occurrence of temporary or permanent $\mathrm{AV}$ conduction issues related to ablation, this finding may guide the approach to ablation, and potentially decrease the risk of inadvertent $\mathrm{AV}$ block during slow pathway ablation in AVNRT. Further studies are recommended to validate this technique in pediatric and congenital heart disease.

\section{References}

1. Ko JK, Deal BJ, Strasburger JF, Benson DW Jr. Supraventricular tachycardia mechanisms and their age distribution in pediatric patients. Am J Cardiol. 1992;69(12):1028-1032.

2. Hintringer F, Hartikainen J, Davies DW, et al. Prediction of atrioventricular block during radiofrequency ablation of the slow pathway of the atrioventricular node. Circulation. 1995;92(12):3490-3496.

3. Delise P, Sitta N, Zoppo F, et al. Radiofrequency ablation of atrioventricular nodal reentrant tachycardia: the risk of intraprocedural, late and long-term atrioventricular block. The Veneto Region multicenter experience. Ital Heart J. 2002;3(12):715-720.

4. Deisenhofer I, Zrenner B, Yin YH, et al. Cryoablation versus radiofrequency energy for the ablation of atrioventricular nodal reentrant tachycardia (the CYRANO Study): Results from a large multicenter prospective randomized trial. Circulation. 2010;122(22):2239-2245.

5. Delise P, Bonso A, Coro L, et al. Pacemapping of the triangle of Koch: a simple method to reduce the risk of atrioventricular block during radiofrequency ablation of atrioventricular node reentrant tachycardia. Pacing Clin Electrophysiol. 2001;24(12): 1725-1731.

6. Delise P, Sitta N, Bonso A, et al. Pace mapping of Koch's triangle reduces risk of atrioventricular block during ablation of atrioventricular nodal reentrant tachycardia. J Cardiovasc Electrophysiol. 2005;16(1):30-35.

7. Blurton DJ, Dubin AM, Chiesa NA, Van Hare GF, Collins KK. Characterizing dual atrioventricular nodal physiology in pediatric patients with atrioventricular nodal reentrant tachycardia. J Cardiovasc Electrophysiol. 2006; 17(6):638-644.

8. Haissaguerre M, Gaita F, Fischer B, et al. Elimination of atrioventricular nodal reentrant tachycardia using discrete slow potentials to guide application of radiofrequency energy. Circulation. 1992;85(6):2162-2175.

9. Jackman WM, Beckman KJ, McClelland JH, et al. Treatment of supraventricular tachycardia due to atrioventricular nodal reentry, by radiofrequency catheter ablation of slowpathway conduction. N Engl J Med. 1992;327(5):313-318.

10. Van Hare GF, Javitz H, Carmelli D, et al; Pediatric Electrophysiology Society. Prospective assessment after pediatric cardiac ablation: Demographics, medical profiles, and initial outcomes. J Cardiovasc Electrophysiol. 2004;15(7):759-770.

11. Collins KK, Dubin AM, Chiesa NA, Avasarala K, Van Hare GF. Cryoablation versus radiofrequency ablation for treatment of pediatric atrioventricular nodal reentrant tachycardia: initial experience with 4-mm cryocatheter. Heart Rhythm. 2006;3(5): 564-570.

12. LaPage MJ, Saul JP, Reed JH. Long-term outcomes for cryoablation of pediatric patients with atrioventricular nodal reentrant tachycardia. Am J Cardiol. 2010;105(8): 1118-1121.

13. Papagiannis J, Papadopoulou K, Rammos S, Katritsis D. Cryoablation versus radiofrequency ablation for atrioventricular nodal reentrant tachycardia in children: Long-term results. Hellenic J Cardiol. 2010;51(2):122-126.

14. Bailin SJ, Korthas MA, Weers NJ, Hoffman CJ. Direct visualization of the slow pathway using voltage gradient mapping: a novel approach for successful ablation of atrioventricular nodal reentry tachycardia. Europace. 2011; 13(8):1188-1194.

15. Malloy L, Law IH, Von Bergen NH. Voltage mapping for slow-pathway visualization and ablation of atrioventricular nodal reentry tachycardia in pediatric and young adult patients. Pediatr Cardiol. 2014;35(1):103-107.

16. Kanter RJ. Pace mapping the fast pathway in patients with atrioventricular nodal reentrant tachycardia: Koch's triangle or the Bermuda Triangle? J Cardiovasc Electrophysiol. 2005;16(1):36-38. 